\title{
Implementation of the Simple Additive Weighting Method in Determining Recipients of Subsidized Food Materials for Poor Families
}

\author{
Kusmanto ${ }^{1}$, Eko Setia Budi2,", Samsir ${ }^{1}$, Elvia Hariska ${ }^{3}$, Guidio Leonarde Ginting ${ }^{3}$ \\ ${ }^{1}$ Informatics Engineering Study Program, Universitas Alwashliyah Labuhanbatu, Rantoprapat, Indonesia \\ ${ }^{2}$ Information System Study Program, Universitas Nusa Mandiri, Jakarta, Indonesia \\ ${ }^{3}$ Informatics Engineering Study Program, Universitas Budi Darma, Medan, Indonesia \\ Email: ${ }^{1}$ kusnabara03@gmail.com, ${ }^{2,}$ eko.etb@ nusamandiri.ac.id, ${ }^{3}$ samsirst111@gmail.com, ${ }^{4}$ hariskae@gmail.com,

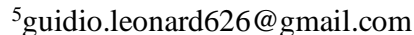 \\ Email Penulis Korespondensi: eko.etb@nusamandiri.ac.id \\ Submitted: 20/12/2021; Accepted: 28/12/2021; Published: 31/12/2021
}

\begin{abstract}
In accordance with the rules that have been set from the Village Office so that the community gets subsidized food, it must comply with the specified criteria. The Village Office will determine who is selected to receive subsidized food and distribute it to poor families. As a tool that can be used to determine someone who is eligible to receive subsidized food, a decision support system is needed. In the decision support system there are several methods, one of which can be used is the SAW (Simple Additive Weighting) method. In this research.
\end{abstract}

Keywords: Decision Support System; Subsidized Foodstuffs; Simple Additive Weighting; SAW Method

\section{INTRODUCTION}

The village office is a center for community services and activities in the village, both in the fields of government, empowerment, development, and coaching[1]. As an example of the service provided by the Village Office is the distribution of subsidized food items that will be received by the poor. In the distribution of subsidized food, data collection is carried out where there are several criteria needed so that the receipt of subsidized food can be carried out optimally.

Indicators in the selection of the poor who get subsidized food have several criteria. These include 1) people who have underprivileged cards, 2) insufficient income, 3) number of dependents, 4) housing, and so on. Until now, the distribution of subsidized feed ingredients provided by the village office has not been fully implemented properly because there are still poor people who have not received it. In order for the distribution of subsidized food to be more objective, of course it requires a tool, namely an information system that aims to process data so as to produce the required information, in the form of rankings for the community. This information system is known as a decision support system (DSS) [2].

Decision support system or often referred to as DSS is an information system that is often used to assist in making a decision. The DSS is usually used by an organization to make decisions about the problems at hand[2]-[5]. In DSS there are ranking methods to facilitate decision making, such as Weighted Product (WP), Simple Additive Weighting (SAW), TOPSIS, ELECTRE, and others [6], [7].

Currently, there are many studies that cover the distribution of subsidized food by implementing a decision support system. Among them are research conducted by R. Mahdalena Simanjorang in 2017 about "Decision Support System for Determining Recipients of Subsidized Food Materials for Poor Families Using the AHP Method at the Mangga Village Office", using this method is expected to help the Mangga Village Office to determine who gets subsidized food by ranking the largest alternative[2]. Masita Handayani in 2017 conducted a study entitled Decision Support System for Determining Raskin Acceptance Using the TOPSIS Method, that in determining Raskin recipients by taking the highest final recipient score[8]. Meanwhile, research conducted by Risa Helilintar in 2016 regarding the Application of the SAW and Fuzzy Methods in the Scholarship Acceptance Decision Support System, that the best alternative value will be chosen [9].

In this study, the authors used the Simple Additive Weighting (SAW) method to determine the distribution of subsidized food at the Galang District Village Office. The results of this study are expected to be able to provide effective decisions that are useful for the Village Office in making decisions on people who are entitled to subsidized food.

\section{RESEARCH METHODOLOGY}

\subsection{Research Stages}

In conducting research at the Village Office, the author carried out several stages of data collection methods used to obtain the data needed by the author, namely:

1. Field Research (Field Research), at this stage the authors conduct research directly. In this initial stage, there are several methods including observation, interviews with village leaders or employees, related to the poor who receive subsidized food. 
2. Library Research, at this stage the author reads books and articles related to previous studies conducted by several experts, as well as to the method used by the author in the decision support system in determining recipients of subsidized food for poor families.

3. Stages of Analysis and Testing, at this stage the author takes several data samples. A total of 20 data samples about poor families who will receive subsidized food, the authors also take a sample of the attributes and criteria that have been set by the Village Office in selecting who is entitled to receive subsidized food. The author will rank using the Simple Additive Weighting (SAW) method to determine 3 families who will receive subsidized snacks.

4. Stages of Results and Research Resume, at this last stage the author will get poor families who are entitled to receive subsidized confectionary materials, also the author can write a research report that he does.

\subsection{Subsidized Foodstuff}

Food is a basic need for human consumption which is obtained from agricultural, vegetable and animal products. Subsidized food is a budget that has been set by the central government for the poor. Subsidized foodstuffs are usually in the form of rice which will later be distributed to the people who are entitled to it[10].

\subsection{Simple Additive Weighting Method}

The Simple Additive Weighting (SAW) method is often also referred to as the weighted addition method which rates each alternative on all existing attributes. The SAW method requires the process of normalizing the decision matrix () to a scale that can be compared with all existing alternative ratings $X_{i j}[11]-[17]$.

SAW Method Stages

1. Preparing the Decision Matrix

Information:

$$
X_{i j}=\left[\begin{array}{cccc}
X_{11} & X_{12} & - & X_{1 n} \\
X_{21} & X_{22} & - & X_{2 n} \\
- & - & - & - \\
X_{m 1} & X_{m 2} & - & X_{m n}
\end{array}\right]
$$

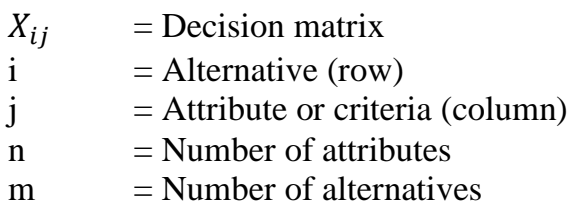

2. Calculating the Normalization Matrix $\left(R_{i j}\right)$

Information:

$$
R_{i j}=\left\{\begin{array}{lc}
\frac{X_{i j}}{\operatorname{Max} X_{i j}} & \text { (benefit attribute) } \\
\frac{\operatorname{Min} X_{i j}}{X_{i j}} & \text { (cost attribute) }
\end{array}\right.
$$

$R_{i j} \quad=$ Normalized matrix

$\operatorname{Max} X_{i j}=$ The highest value in the $\mathrm{j}$-th column

$\operatorname{Min} X_{i j}=$ The lowest value in the $\mathrm{j}$-th column

$X_{i j} \quad=$ Decision matrix

3. Calculating Preference Value $\left(V_{i}\right)$

At this final stage to determine the rating value of each alternative. A larger value indicates that the alternative is more selected. $\left(V_{i}\right)\left(A_{i}\right)$

Information:

$$
V_{i}=\sum_{j=1}^{n} W_{j} R_{i j}
$$

$V_{i} \quad=$ preference value

$W_{j} \quad=$ Weight

$R_{i j} \quad=$ Normalized matrix

$\mathrm{j}=$ Criteria/attributes

$\mathrm{n} \quad=$ Number of criteria/attributes

\section{RESULTS AND DISCUSSION}

One of the problems faced in determining who is entitled to receive subsidized food in the village is the large number of people who register to get benefits from the government. Therefore, the Village Head and Village Staff need a ranking of the people who have registered, by looking for the best alternative. 
Based on this problem, the author makes a Decision Support System for Determining the Acceptance of Subsidized Foodstuffs for Poor Families with the SAW Method at the Village Office. The following are the criteria for receiving subsidized food for poor families:

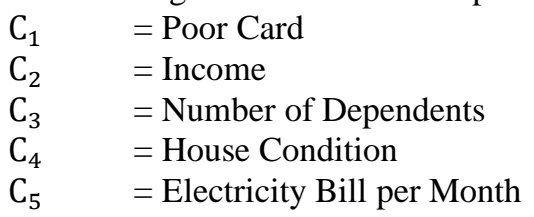

And as an alternative, the author uses 20 data samples, namely:

$\mathrm{A}_{1} \quad=$ Maruti

$\mathrm{A}_{2} \quad=$ Saidi

$\mathrm{A}_{3} \quad=$ Sri

$\mathrm{A}_{4} \quad=$ Praise

$\mathrm{A}_{5} \quad=$ Lucy

$\mathrm{A}_{6} \quad=$ poniran

$\mathrm{A}_{7} \quad=$ Paiman

$\mathrm{A}_{8} \quad=$ Parno

$\mathrm{A}_{9} \quad=$ Mukiran

$\mathrm{A}_{10}=$ Zum

$\mathrm{A}_{11}=$ Tukiyo

$\mathrm{A}_{12}=$ Tumin

$\mathrm{A}_{13}=$ Heri

$\mathrm{A}_{14}=$ Ngatijo

$\mathrm{A}_{15}=$ Supri

$\mathrm{A}_{16}=$ Mujiah

$\mathrm{A}_{17} \quad=$ Dian

$\mathrm{A}_{18}=$ Ono

$\mathrm{A}_{19} \quad=$ Arju

$\mathrm{A}_{20}=$ Dona

In this SAW method, it is necessary to determine the attributes of each criterion and choose its weight. This is to select the use of the weighted value of each criterion for the selection process. Decision making gives weight to each criterion viz.

Table 1. Criteria for Accepting Subsidized Foodstuffs

\begin{tabular}{lccc}
\hline \multicolumn{1}{c}{ Criteria } & Variable & Type & Weight \\
\hline Poor Card & $\mathrm{C}_{1}$ & Benefits & $25 \%$ \\
Income & $\mathrm{C}_{2}$ & Benefits & $20 \%$ \\
The number of dependents & $\mathrm{C}_{3}$ & Benefits & $20 \%$ \\
Home Condition & $\mathrm{C}_{4}$ & Benefits & $20 \%$ \\
Electricity Bill per Month & $\mathrm{C}_{5}$ & Benefits & $15 \%$ \\
\hline
\end{tabular}

Table 2. Weighting Criteria $\mathrm{C} 1, \mathrm{C} 4$

\begin{tabular}{clc}
\hline Criteria & \multicolumn{1}{c}{ Score } & Weight \\
\hline Poor Card & Do not have & 2 \\
& Have & 3 \\
\hline \multirow{2}{*}{ Home Condition } & Rumbiah Wall, Earth Floor & 5 \\
& Plank Wall, Plank Floor & 4 \\
& Cement Wall, Earth Floor & 3 \\
& Cement Wall, Cement Floor & 2 \\
& Cement Wall, Ceramic Floor & 1 \\
\hline
\end{tabular}

The following table 3 is the data that has registered to get subsidized food in the village,

Table 3. Data on Prospective Subsidized Food Recipients

\begin{tabular}{|c|c|c|c|c|c|}
\hline \multirow{2}{*}{ Alternative } & \multicolumn{5}{|c|}{ Criteria } \\
\hline & $\mathrm{C} 1$ & $\mathrm{C} 2$ & $\mathrm{C} 3$ & $\mathrm{C} 4$ & $\mathrm{C} 5$ \\
\hline Maruti & Have & 300,000 & 3 & Cement Wall, Cement Floor & 50,000 \\
\hline Saidi & Have & 200,000 & 5 & Cement Wall, Earth Floor & 50,000 \\
\hline Sri & Do not have & 700,000 & 1 & Cement Wall, Ceramic Floor & 90,000 \\
\hline
\end{tabular}


Building of Informatics, Technology and Science (BITS)

Volume 3, No 3, Desember 2021 Page: 384-392

ISSN 2684-8910 (media cetak)

ISSN 2685-3310 (media online)

DOI 10.47065/bits.v3i3.1097

\begin{tabular}{llrllr}
\hline Alternative & \multicolumn{1}{c}{ C1 } & \multicolumn{1}{c}{ C2 } & C3 & Criteria & \multicolumn{1}{c}{ C4 5} \\
Praise & Do not have & $1,000,000$ & 1 & Cement Wall, Cement Floor & 50,000 \\
Lucy & Do not have & $1,500,000$ & 5 & Cement Wall, Ceramic Floor & 120,000 \\
poniran & Do not have & $1,000,000$ & 2 & Cement Wall, Ceramic Floor & 200,000 \\
Paiman & Do not have & 500,000 & 2 & Cement Wall, Ceramic Floor & 130,000 \\
Parno & Do not have & 400,000 & 2 & Cement Wall, Cement Floor & 80,000 \\
carving & Do not have & $1,500,000$ & 3 & Cement Wall, Cement Floor & 50,000 \\
Zum & Have & $1,000,000$ & 6 & Cement Wall, Earth Floor & 50,000 \\
Tukiyo & Do not have & 800,000 & 3 & Cement Wall, Cement Floor & 50,000 \\
Tumin & Do not have & 700,000 & 2 & Cement Wall, Cement Floor & 50,000 \\
Harry & Do not have & 800,000 & 2 & Cement Wall, Cement Floor & 70,000 \\
Ngatijo & Have & 500,000 & 4 & Cement Wall, Earth Floor & 50,000 \\
supri & Do not have & 700,000 & 1 & Cement Wall, Ceramic Floor & 100,000 \\
Mujiah & Have & 600,000 & 3 & Cement Wall, Cement Floor & 90,000 \\
Dian & Do not have & $1,000,000$ & 1 & Cement Wall, Ceramic Floor & 120,000 \\
Ono & Do not have & 400,000 & 2 & Cement Wall, Cement Floor & 120,000 \\
Arju & Do not have & $1,500,000$ & 3 & Cement Wall, Earth Floor & 100,000 \\
Dona & Do not have & 800,000 & 2 & Cement Wall, Cement Floor & 120,000 \\
\hline
\end{tabular}

From table 3 , it produces a match rating between the alternatives and the criteria.

Table 4. Match Rating

\begin{tabular}{cccccc}
\hline \multirow{2}{*}{ Alternative } & \multicolumn{5}{c}{ Criteria } \\
& C1 & C2 & C3 & C4 & C5 \\
\hline A1 & 3 & 300,000 & 3 & 2 & 50,000 \\
A2 & 3 & 200,000 & 5 & 3 & 50,000 \\
A3 & 2 & 700,000 & 1 & 1 & 90,000 \\
A4 & 2 & $1,000,000$ & 1 & 2 & 50,000 \\
A5 & 2 & $1,500,000$ & 5 & 1 & 120,000 \\
A6 & 2 & $1,000,000$ & 2 & 1 & 200,000 \\
A7 & 2 & 500,000 & 2 & 1 & 130,000 \\
A8 & 2 & 400,000 & 2 & 2 & 80,000 \\
A9 & 2 & $1,500,000$ & 3 & 2 & 50,000 \\
A10 & 3 & $1,000,000$ & 6 & 3 & 50,000 \\
A11 & 2 & 800,000 & 3 & 2 & 50,000 \\
A12 & 2 & 700,000 & 2 & 2 & 50,000 \\
A13 & 2 & 800,000 & 2 & 2 & 70,000 \\
A14 & 3 & 500,000 & 4 & 3 & 50,000 \\
A15 & 2 & 700,000 & 1 & 1 & 100,000 \\
A16 & 3 & 600,000 & 3 & 2 & 90,000 \\
A17 & 2 & $1,000,000$ & 1 & 1 & 120,000 \\
A18 & 2 & 400,000 & 2 & 2 & 120,000 \\
A19 & 2 & $1,500,000$ & 3 & 3 & 100,000 \\
A20 & 2 & 800,000 & 2 & 2 & 120,000 \\
\hline
\end{tabular}

The next stage of the Saw method is to make a decision matrix from the compatibility rating that has been generated.

1. Preparing the Decision Matrix 
2. Calculating Normalized Matrices $\left(\mathrm{R}_{\mathrm{ij}}\right)$

For Criteria $\mathrm{C}_{1}$ (Benefit) by using the equation (2)

$\mathrm{R}_{1.1}=3 / 3=1$

$\mathrm{R}_{2.1}=3 / 3=1$

$\mathrm{R}_{3.1}=2 / 3=0.667$

$\mathrm{R}_{4.1}=2 / 3=0.667$

$\mathrm{R}_{5.1}=2 / 3=0.667$

$\mathrm{R}_{6.1}=2 / 3=0.667$

$\mathrm{R}_{7.1}=2 / 3=0.667$

$\mathrm{R}_{8.1}=2 / 3=0.667$

$\mathrm{R}_{9.1}=2 / 3=0.667$

$\mathrm{R}_{10.1}=3 / 3=1$

$\mathrm{R}_{11.1}=2 / 3=0.667$

$\mathrm{R}_{12.1}=2 / 3=0.667$

$\mathrm{R}_{13.1}=2 / 3=0.667$

$\mathrm{R}_{14.1}=3 / 3=1$

$\mathrm{R}_{15.1}=2 / 3=0.667$

$\mathrm{R}_{16.1}=3 / 3=1$

$\mathrm{R}_{17.1}=2 / 3=0.667$

$\mathrm{R}_{18.1}=2 / 3=0.667$

$\mathrm{R}_{19.1}=2 / 3=0.667$

$\mathrm{R}_{20.1}=2 / 3=0.667$

For Criteria $\mathrm{C}_{2}$ (Benefit) by using the equation (2)

$\begin{array}{ll}\mathrm{R}_{1.2}=300,000 / 1,500,000 & =0.2 \\ \mathrm{R}_{2.2}=200,000 / 1,500,000 & =0.133 \\ \mathrm{R}_{3.2}=700,000 / 1,500,000 & =0.467 \\ \mathrm{R}_{4.2}=1,000,000 / 1,500,000 & =0.667 \\ \mathrm{R}_{5.2}=1,500,000 / 1,500,000 & =1 \\ \mathrm{R}_{6.2}=1,000,000 / 1,500,000 & =0.667 \\ \mathrm{R}_{7.2}=500,000 / 1,500,000 & =0.333 \\ \mathrm{R}_{8.2}=400,000 / 1,500,000 & =0.267 \\ \mathrm{R}_{9.2}=1,500,000 / 1,500,000 & =1 \\ \mathrm{R}_{10.2}=1,000,000 / 1,500,000 & =0.667 \\ \mathrm{R}_{11.2}=800,000 / 1,500,000 & =0.533 \\ \mathrm{R}_{12.2}=700,000 / 1,500,000 & =0.467 \\ \mathrm{R}_{13.2}=800,000 / 1,500,000 & =0.533 \\ \mathrm{R}_{14.2}=500,000 / 1,500,000 & =0.333\end{array}$


Building of Informatics, Technology and Science (BITS)

Volume 3, No 3, Desember 2021 Page: 384-392

ISSN 2684-8910 (media cetak)

ISSN 2685-3310 (media online)

DOI 10.47065/bits.v3i3.1097

$\begin{array}{ll}\mathrm{R}_{15.2}=700,000 / 1,500,000 & =0.467 \\ \mathrm{R}_{16.2}=600,000 / 1,500,000 & =0.4 \\ \mathrm{R}_{17.2}=1,000,000 / 1,500,000 & =0.667 \\ \mathrm{R}_{18.2}=400,000 / 1,500,000 & =0.267 \\ \mathrm{R}_{19.2}=1,500,000 / 1,500,000 & =1 \\ \mathrm{R}_{20.2}=800,000 / 1,500,000 & =0.533\end{array}$

For Criteria $\mathrm{C}_{3}$ (Benefit) by using the equation (2)

$\mathrm{R}_{1.3}=3 / 6=0.6$

$\mathrm{R}_{2.3}=5 / 6=0.833$

$\mathrm{R}_{3.3}=1 / 6=0.167$

$\mathrm{R}_{4.3}=1 / 6=0.167$

$\mathrm{R}_{5.3}=5 / 6=0.833$

$\mathrm{R}_{6.3}=2 / 6=0.333$

$\mathrm{R}_{7.3}=2 / 6=0.333$

$\mathrm{R}_{8.3}=2 / 6=0.333$

$\mathrm{R}_{9.3}=3 / 6=0.6$

$\mathrm{R}_{10.3}=6 / 6=1$

$\mathrm{R}_{11.3}=3 / 6=0.6$

$\mathrm{R}_{12.3}=2 / 6=0.333$

$\mathrm{R}_{13.3}=2 / 6=0.333$

$\mathrm{R}_{14.3}=4 / 6=0.667$

$\mathrm{R}_{15.3}=1 / 6=0.167$

$\mathrm{R}_{16.3}=3 / 6=0.6$

$\mathrm{R}_{17.3}=1 / 6=0.167$

$\mathrm{R}_{18.3}=2 / 6=0.333$

$\mathrm{R}_{19.3}=3 / 6=0.6$

$\mathrm{R}_{20.3}=2 / 6=0.333$

For Criteria $\mathrm{C}_{4}$ (Benefit) by using the equation (2)

$\mathrm{R}_{1.4}=2 / 3=0.667$

$\mathrm{R}_{2.4}=3 / 3=1$

$\mathrm{R}_{3.4}=1 / 3=0.333$

$\mathrm{R}_{4.4}=2 / 3=0.667$

$\mathrm{R}_{5.4}=1 / 3=0.333$

$\mathrm{R}_{6.4}=1 / 3=0.333$

$\mathrm{R}_{7.4}=1 / 3=0.333$

$\mathrm{R}_{8.4}=2 / 3=0.667$

$R_{9.4}=2 / 3=0.667$

$\mathrm{R}_{10.4}=3 / 3=1$

$\mathrm{R}_{11.4}=2 / 3=0.667$

$\mathrm{R}_{12.4}=2 / 3=0.667$

$\mathrm{R}_{13.4}=2 / 3=0.667$

$\mathrm{R}_{14.4}=3 / 3=1$

$R_{15.4}=1 / 3=0.333$

$\mathrm{R}_{16.4}=2 / 3=0.667$

$\mathrm{R}_{17.4}=1 / 3=0.333$

$\mathrm{R}_{18.4}=2 / 3=0.667$

$\mathrm{R}_{19.4}=3 / 3=1$

$R_{20.4}=2 / 3=0.667$

For Criteria $\mathrm{C}_{5}$ (Benefit) by using the equation (2)

$\mathrm{R}_{1.5}=50,000 / 200,000 \quad=0.23$

$\mathrm{R}_{2.5}=50,000 / 200,000 \quad=0.23$

$\mathrm{R}_{3.5}=90,000 / 200,000 \quad=0.45$

$\mathrm{R}_{4.5}=50,000 / 200,000=0.25$

$\mathrm{R}_{5.5}=120,000 / 200,000=0.6$

$\mathrm{R}_{6.5}=200,000 / 200,000=1$

$\mathrm{R}_{7.5}=130.00 / 200,000=0.65$

$\mathrm{R}_{8.5}=80,000 / 200,000 \quad=0.4$ 


$$
\begin{array}{ll}
\mathrm{R}_{9.5}=50,000 / 200,000 & =0.25 \\
\mathrm{R}_{10.5}=50,000 / 200,000 & =0.25 \\
\mathrm{R}_{11.5}=50,000 / 200,000 & =0.25 \\
\mathrm{R}_{12.5}=50,000 / 200,000 & =0.25 \\
\mathrm{R}_{13.5}=70,000 / 200,000 & =0.35 \\
\mathrm{R}_{14.5}=50,000 / 200,000 & =0.25 \\
\mathrm{R}_{15.5}=100.00 / 200,000 & =0.5 \\
\mathrm{R}_{16.5}=90,000 / 200,000 & =0.45 \\
\mathrm{R}_{17.5}=120,000 / 200,000 & =0.6 \\
\mathrm{R}_{18.5}=120,000 / 200,000 & =0.6 \\
\mathrm{R}_{19.5}=100,000 / 200,000 & =0.5 \\
\mathrm{R}_{20.5}=120,000 / 12000 & =0.6
\end{array}
$$

The results obtained for the normalized matrix are: $\left(\mathrm{R}_{\mathrm{ij}}\right)$

$\mathrm{R}_{\mathrm{ij}}=\left[\begin{array}{ccccc}1 & 0,2 & 0,6 & 0,667 & 0,23 \\ 1 & 0,133 & 0,833 & 1 & 0,23 \\ 0,667 & 0,467 & 0.167 & 0,333 & 0,45 \\ 0,667 & 0,667 & 0.167 & 0,667 & 0,25 \\ 0,667 & 1 & 0,833 & 0,333 & 0,6 \\ 0,667 & 0,667 & 0,333 & 0,333 & 1 \\ 0,667 & 0,333 & 0,333 & 0,333 & 0,65 \\ 0,667 & 0,267 & 0,333 & 0,667 & 0,4 \\ 0,667 & 1 & 0,6 & 0,667 & 0,25 \\ 1 & 0,667 & 1 & 1 & 0,25 \\ 0,667 & 0,533 & 0,6 & 0,667 & 0,25 \\ 0,667 & 0,467 & 0,333 & 0,667 & 0,25 \\ 0,667 & 0,533 & 0,333 & 0,667 & 0,35 \\ 1 & 0,333 & 0,167 & 1 & 0,25 \\ 0,667 & 0,467 & 0,167 & 0,333 & 0,5 \\ 1 & 0,4 & 0,6 & 0,667 & 0,45 \\ 0,667 & 0,667 & 0,167 & 0,333 & 0,6 \\ 0,667 & 0,267 & 0,333 & 0,667 & 0,6 \\ 0,667 & 1 & 0,6 & 1 & 0,5 \\ 0,667 & 0,533 & 0,333 & 0,667 & 0,6\end{array}\right]$

3. Finding the Preference Value by using the formula for equation (3) $\left(V_{i}\right)$

$$
\begin{array}{lr}
\mathrm{V}_{1}=\sum(0,25 * 1)+(0,2 * 0,2)+(0,2 * 0,6)+(0,2 * 0,667)+(0,15 * 0,23) & =0.557 \\
\mathrm{~V}_{2}=\sum(0,25 * 1)+(0,2 * 0,133)+(0,2 * 0,833)+(0,2 * 1)+(0,15 * 0,23) & =0.910 \\
\mathrm{~V}_{3}=\sum(0,25 * 0,667)+(0,2 * 0,467)+(0,2 * 0,167)+(0,2 * 0,333)+(0,15 * 0,45) & =0.602 \\
\mathrm{~V}_{4}=\sum(0,25 * 0,667)+(0,2 * 0,667)+(0,2 * 0,167)+(0,2 * 0,667)+(0,15 * 0,25) & =0.698 \\
\mathrm{~V}_{5}=\sum(0,25 * 0,667)+(0,2 * 1)+(0,2 * 0,883)+(0,2 * 0,333)+(0,15 * 0,6) & =0.581 \\
\mathrm{~V}_{6}=\sum(0,25 * 0,667)+(0,2 * 0,667)+(0,2 * 0,333)+(0,2 * 0,333)+(0,15 * 1) & =0.461 \\
\mathrm{~V}_{7}=\sum(0,25 * 0,667)+(0,2 * 0,333)+(0,2 * 0,333)+(0,2 * 0,333)+(0,15 * 0,65) & =0.478 \\
\mathrm{~V}_{8}=\sum(0,25 * 0,667)+(0,2 * 0,267)+(0,2 * 0,333)+(0,2 * 0,667)+(0,15 * 0,4) & =0.656 \\
\mathrm{~V}_{9}=\sum(0,25 * 0,667)+(0,2 * 1)+(0,2 * 0,6)+(0,2 * 0,667)+(0,15 * 0,25) & =0.820 \\
\mathrm{~V}_{10}=\sum(0,25 * 1)+(0,2 * 0,667)+(0,2 * 1)+(0,2 * 1)+(0,15 * 0,25) & =0.562 \\
\mathrm{~V}_{11}=\sum(0,25 * 0,667)+(0,2 * 0,533)+(0,2 * 0,6)+(0,2 * 0,667)+(0,15 * 0,25) & =0.495 \\
\mathrm{~V}_{12}=\sum(0,25 * 0,667)+(0,2 * 0,467)+(0,2 * 0,333)+(0,2 * 0,667)+(0,15 * 0,25) & =0.523 \\
\mathrm{~V}_{13}=\sum(0,25 * 0,667)+(0,2 * 0,533)+(0,2 * 0,333)+(0,2 * 0,667)+(0,15 * 0,35) & =0.586 \\
\mathrm{~V}_{14}=\sum(0,25 * 1)+(0,2 * 0,333)+(0,2 * 0,167)+(0,2 * 1)+(0,15 * 0,25) & =0.433 \\
\mathrm{~V}_{15}=\sum(0,25 * 0,667)+(0,2 * 0,467)+(0,2 * 0,167)+(0,2 * 0,333)+(0,15 * 0,5) &
\end{array}
$$




$$
\begin{array}{lll}
\mathrm{V}_{16}=\sum(0,25 * 1)+(0,2 * 0,4)+(0,2 * 0,6)+(0,2 * 0,667)+(0,15 * 0,45) & =0.650 \\
\mathrm{~V}_{17}=\sum(0,25 * 0,667)+(0,2 * 0,667)+(0,2 * 0,167)+(0,2 * 0,333)+(0,15 * 0,6) & =0.488 \\
\mathrm{~V}_{18}=\sum(0,25 * 0,667)+(0,2 * 0,267)+(0,2 * 0,333)+(0,2 * 0,667)+(0,15 * 0,6) & =0.508 \\
\mathrm{~V}_{19}=\sum(0,25 * 0,667)+(0,2 * 1)+(0,2 * 0,6)+(0,2 * 1)+(0,15 * 0,5) & =0.761 \\
\mathrm{~V}_{20}=\sum(0,25 * 0,667)+(0,2 * 0,533)+(0,2 * 0,333)+(0,2 * 0,667)+(0,15 * 0,6) & =0.561
\end{array}
$$

From the results of the preference values above, the final results are:

Table 5. The final result

\begin{tabular}{cccc}
\hline Alternative & Name & Vi & Rating \\
\hline A1 & Maruti & 0.557 & 12 \\
A2 & Saidi & 0.910 & 1 \\
A3 & Sri & 0.425 & 20 \\
A4 & Praise & 0.602 & 7 \\
A5 & Lucy & 0.698 & 4 \\
A6 & poniran & 0.581 & 9 \\
A7 & Paiman & 0.461 & 18 \\
A8 & Parno & 0.478 & 17 \\
A9 & carving & 0.656 & 5 \\
A10 & Zum & 0.820 & 2 \\
A11 & Tukiyo & 0.562 & 10 \\
A12 & Tumin & 0.495 & 15 \\
A13 & Harry & 0.523 & 13 \\
A14 & Ngatijo & 0.586 & 8 \\
A15 & supri & 0.433 & 19 \\
A16 & Mujiah & 0.650 & 6 \\
A17 & Dian & 0.488 & 16 \\
A18 & Ono & 0.508 & 4 \\
A19 & Arju & 0.761 & 3 \\
A20 & Dona & 0.561 & 11 \\
\hline
\end{tabular}

From the final results above, it can be recommended that the 5 families with the highest scores receive subsidized food at the Village Office, namely:
1. Saidi $\left(\mathrm{A}_{2}\right)$
2. $\operatorname{Zum}\left(\mathrm{A}_{10}\right)$
3. $\operatorname{Arju}\left(A_{19}\right)$
4. $\operatorname{Lucy}\left(\mathrm{A}_{5}\right)$
5. carving $\left(A_{9}\right)$

\section{CONCLUSION}

From the results and discussion, it can be concluded that the decision support system using the simple additive weighting method is very helpful in determining the receipt of subsidized food at the Village Office because many people have volunteered as alternatives so that by using this method the Village Head and village staff can decide one or the other. the best alternative would be to receive subsidized food from the Village Office.

\section{REFERENCES}

[1] A. Rusmayanti, "Sistem Informasi Pengelolaan Keuangan Pada Desa Ngadirejan," J. Speed - Sentra Penelit. Eng. dan Edukasi, vol. 6, no. 2, pp. 35-39, 2014.

[2] R. M. Simanjorang, H. D. Hutahaean, H. T. Sihotang, and T. Informatika, "Sistem pendukung keputusan penentuan penerima bahan pangan bersubsidi untuk keluarga miskin dengan metode ahp pada kantor kelurahan mangga," J. Inform. Pelita Nusant., vol. 2, no. 1, pp. 22-31, 2017.

[3] T. Limbong et al., Sistem Pendukung Keputusan: Metode \& Implementasi. Medan: Yayasan Kita Menulis, 2020.

[4] D. Nofriansyah, Multi Criteria Decision Making (MCDM) Pada Sistem Pendukung Keputusan. Yogyakarta: CV.Budi Utama, 2017.

[5] Efraim Turban and Jay E. Aronson, Decision Support System and Intelligent Systems. 2001.

[6] M. K. Muhammad Decky Andani, Yeka Hendriyani, S.Kom., "PERANCANGAN SISTEM PENDUKUNG KEPUTUSAN PENENTUAN PENERIMA BANTUAN DAERAH BERDASARKAN TARAF HIDUP MASYARAKAT 
MENGGUNAKAN METODE SIMPLE ADDITIVE WEIGHTING (SAW) (STUDI KASUS : DESA MEDAN JAYA) Muhammad," J. Vokasional Tek. Elektron. dan Inform., vol. 6, no. 2, 2018.

[7] Mesran, E. P. Sumantri, Supriyanto, S. H. Sahir, and N. K. Daulay, "Implementation of Technique for Order Preference by Similarity to Ideal Solution (TOPSIS) in Recommendations for New Position in Companies," Int. J. Inf. Syst. Technol., vol. 4, no. 2, pp. 661-669, 2021.

[8] M. Handayani, "Sistem pendukung keputusan penentuan penerimaan raskin di menggunakan metode topsis," J. Teknol. Inf., vol. 1, pp. 54-60, 2017.

[9] H. A. F. (Magister T. I. S. A. Y. Helilintar, Risa, Wing Wahyu Winarno, "Penerapan Metode SAW dan Fuzzy Dalam Sistem Pendukung Keputusan Penerimaan Beasiswa in Decision Support System Scholarship," Citec J., vol. 3, no. 2, pp. 89-101, 2016.

[10] D. W. Prabowo and J. M. I. R. R. N. . J. P. dwi_wp@yahoo. c. Pusat Kebijakan Perdagangan Dalam Negeri, BP2KP, Kementerian Perdagangan - RI, "PENGELOMPOKAN KOMODITI BAHAN PANGAN POKOK DENGAN METODE ANALYTICAL HIERARCHY PROCESS Classification of Staple Food Commodity Using Analytical Hierarchy Process," pp. 163-182, 2014.

[11] R. S. P. L. Tri Susilowati, "SISTEM PENDUKUNG KEPUTUSAN SELEKSI PENERIMAAM CALON SISWA BARU PADA SMA MUHAMADIYAH 1 PRINGSEWU DENGAN," pp. 12-21.

[12] I. J. T. Situmeang, S. Hummairoh, S. M. Harahap, and Mesran, "Application of SAW ( Simple Additive Weighting ) for the Selection of Campus Ambassadors," IJICS (International J. Informatics Comput. Sci., vol. 5, no. 1, pp. 21-28, 2021.

[13] M. R. Ramadhan, M. K. Nizam, and Mesran, "Penerapan Metode SAW (Simple Additive Weighting) Dalam Pemilihan Siswa-Siswi Berprestasi Pada Sekolah SMK Swasta Mustafa," TIN Terap. Inform. Nusant., vol. 1, no. 9, pp. 459-471, 2021.

[14] R. Y. Simanullang, Melisa, and Mesran, "Sistem Pendukung Keputusan Penerima Bantuan Covid-19 Menggunakan Metode Simple Additive Weighting (SAW)," TIN Terap. Inform. Nusant., vol. 1, no. 9, pp. 2-9, 2021.

[15] S. K. Simanullang and A. G. Simorangkir, "Sistem Pendukung Keputusan Penerimaan Calon Karyawan Menggunakan Metode Simple Additive Weighting," TIN Terap. Inform. Nusant., vol. 1, no. 9, pp. 472-478, 2021.

[16] S. H. Sahir, R. Rosmawati, and K. Minan, "Simple Additive Weighting Method to Determining Employee Salary Increase Rate," Int. J. Sci. Res. Sci. Technol., vol. 3, no. 8, pp. 42-48, 2017.

[17] R. T. Utami, D. Andreswari, and Y. Setiawan, "Implementasi Metode Simple Additive Weighting (SAW) Dengan Pembobotan Rank Order Centroid (ROC) Dalam Pengambilan Keputusan Untuk Seleksi Pengguna Jasa Leasing Mobil (Studi Kasus: PT. Multindo Auto Finance Cabang Bengkulu)," J. Rekursif, vol. 4, no. 2, pp. 209-221, 2016. 